\title{
Proof of Stronger Goldbach Conjecture
}

\author{
Wildan Waleed Mahmood . \\ Dept. of Mathematics, College of Education, University of Wasit, Iraq \\ wldan@uowasit.edu.iq
}

Recived : $214 \backslash 2018$

Revised : $3 \backslash 5 \backslash 2018$

Accepted : $23 \backslash 5 \backslash 2018$

Available online : $\quad 5 / 8 / 2018$

DOI: $10.29304 / j q c m .2018 .10 .3 .400$

\begin{abstract}
The stronger (binary) Goldbach conjecture expresses that "every even integer greater than or equal to 4 can be written as a sum of two odd prime numbers". The introduce paper demonstrates this conjecture by proven that there exists a positive integer $a$ for each integer number $n \geq 4$, such that $n-a$ and $n+a$ are simultaneously primes.
\end{abstract}

Keywords: Number theory; Primes and their distribution; Goldbach conjecture.

Mathematics Subject Classification : 11P32 
Wildan .W

\section{Introduction.}

The issue under thought had its beginning in a letter composed by Goldbach to Euler in 1742 [1]. He expounded on his thought to the celebrated mathematician Euler, who at initially addressed the letter with some neglect, viewing the outcome as minor. That wasn't very wise of Euler: the "Goldbach conjecture", as it's turned out to be known, remains unproven 'til today which has been verified and it is currently known to be align to 4 . $10^{14,}$ (see [2]). This guess suggests the guess that "all odd numbers greater than 7 are the sum of three odd primes", which is alluded to today differently as the odd Goldbach guess, or the ternary Goldbach guess. While the feeble Goldbach guess seems to have been at last demonstrated in 2013 by Helfgott [3], [4].

We should attempt with a few cases, for instance:

$4=2+2$,

$6=3+3$

$10=3+7$,

$12=5+7$,

$14=7+7$,

$16=3+13$,

$18=7+11$.

See that the cases are not one of a kind, for example $14=3+11=7+7$.

\section{The proof of stronger Goldbach conjecture.}

The proof is very simple after we prove the following theorem :

Theorem (2.1) For each $n \in \mathbb{Z}, n \geq 4, \exists a \in$ $\mathbb{Z}^{+}$, such that $(n-a)$ and $(n+a)$ are simultaneously primes.

Example (2.2) We first verify that there exists such a positive integer number $a$, for $4 \leq n \leq 71$, and the results were recorded as table 1 .

\begin{tabular}{|l|l|l|l|}
\hline$n$ & \multicolumn{1}{|c|}{$a$} & $n$ & \multicolumn{1}{c|}{$a$} \\
\hline 4 & 1 & 38 & $9,15,21,23,33,35$ \\
\hline 5 & 2 & 39 & $2,8,20,22,28,32,34$ \\
\hline 6 & 1 & 40 & $3,21,27,33$ \\
\hline 7 & 4 & 41 & $12,18,30,38$ \\
\hline 8 & 3,5 & 42 & $1,5,11,19,25,29,31,37$ \\
\hline 9 & 2,4 & 43 & $24,30,36,40$ \\
\hline 10 & 3,7 & 44 & $3,15,27,39$ \\
\hline 11 & 6,8 & 45 & $2,8,14,16,22,26,28,34,38$ \\
\hline 12 & $1,5,7$ & 46 & $15,33,43$ \\
\hline 13 & 6,10 & 47 & $6,24,36,42$ \\
\hline 14 & 3,9 & 48 & $5,11,19,25,31,35,41$ \\
\hline 15 & $2,4,8$ & 49 & $12,18,30$ \\
\hline 16 & 3,13 & 50 & $3,9,21,33,39,47$ \\
\hline 17 & $6,12,14$ & 51 & $8,10,20,22,28,32,38,46$ \\
\hline 18 & $1,5,11,13$ & 52 & $9,15,21,45,49$ \\
\hline 19 & 12 & 53 & $6,30,36,48,50$ \\
\hline 20 & $3,9,17$ & 54 & $7,13,17,25,35,43,47,51$ \\
\hline 21 & $2,8,10,16$ & 55 & $12,18,24,42,48,52$ \\
\hline 22 & $9,15,19$ & 56 & $3,15,27,33,45,51,53$ \\
\hline 23 & 6,18 & 57 & $4,10,14,16,26,40,44,46$, \\
\hline 24 & $5,7,13,17,19$ & 58 & $15,21,39,45,51,55$ \\
\hline 25 & $6,12,18,22$ & 59 & $12,30,42,48,54$ \\
\hline 26 & 3,15 & 60 & $1,7,13,19,23,29,37,41,43$, \\
& & 61 & $18,49,48$ \\
\hline 27 & $4,10,14,16,20$ & 62 & $9,21,39,45,51$ \\
\hline 28 & $9,15,25$ & 63 & $10,16,20,26,34,40,44,46$, \\
\hline 29 & $12,18,24$ & 64 & $3,33,45$ \\
\hline 30 & $1,7,11,13,17,23$ & 65 & $6,18,24,36,42,48$ \\
\hline 31 & 12,28 & 66 & $5,7,13,23,35,37,43,47,61$ \\
\hline 32 & $9,15,21,27,29$ & 67 & $6,30,36,60,64$ \\
\hline 33 & $4,10,14,20,26,28$ & 68 & $15,21,39,45,63$ \\
\hline 34 & 3,27 & 69 & $2,10,28,32,38,40,58,62$ \\
\hline 35 & $6,12,18,24,32$ & 70 & $3,9,27,33,39,57,67$ \\
\hline 36 & $5,7,11,17,23,25,31$ & 71 & $12,18,30,42,60,66,68$ \\
\hline 37 & $6,24,30,34$ & & \\
\hline & & & \\
\hline
\end{tabular}

Table1. Checking conjecture $1,4 \leq n \leq 71$. 
Remark (2.3) From table 1, we observe that notes:

i. If $n$ is even (odd) then $a$ is odd (even). This is always true since we want $n+a$ odd prime number.

ii. Since we want $n-a$ prime number, we have $n-a>0$. Therefor, always we have $a<n$.

iii. For every even integer no. $n$ : $a$ can takes the values $n-1$, $n-3, n-5, \ldots, n-(n-1)$.

Also for every odd integer no. $n$ :

$a$ can takes the values $n-1$, $n-3, n-5, \ldots, n-(n-2)$.

iv. we get for every integer $n \geq 4, a=$ $n-p$, where $p$ is prime number, $3 \leq p \leq(n-1)$ when $n$ is even, and $3 \leq p \leq(n-2)$ when $n$ is odd, $n-a=n-(n-p)=p$.

v. Let $S=\{1,3,5,7, \ldots, n-1\}$. And $T=\{2,4,6,8, \ldots, n-1\}$.

It is easy to show that two cases:

1. Let $a \in S$. For every positive even integer $n, n+a$ is odd number such that $2 n-(n-1) \leq n+$ $a \leq 2 n-1$.

2. Let $a \in T$. For every positive odd integer $n, n+a$ is odd number Such that

$$
\begin{aligned}
& 2 n-(n-2) \leq n+a \leq 2 n- \\
& 1, \quad \text { in both }
\end{aligned}
$$

in both

cases there exists $a$ such that $n+a$ is prime.

vi. From the above notes, we observe that for every integer $n \geq 4$, there exists positive integers $a$ such that $n+a$ is prime and $n-a$ is prime.

Now we are looking for an integer $a$ makes $n+a$ and $n-a$ are

Simultaneously primes.

If $n-a=p$ then

$n+a=n+n-p=2 n-p$, where $p$ is prime number such that $3 \leq p \leq$ $(n-1)$, if $n$ is even and $3 \leq p \leq$ $(n-2)$, if $n$ is odd.

Therefor we want to show that $2 n-p$ is prime for some $p$.

\section{Example (2.4)}

Consider the following example, when $n$ odd number. We define a function $f(x): A \rightarrow B$, such that

$A=\{1,3,5, \ldots, n-2\}$,

$B=\{2 n-1,2 n-3,2 n-5, \ldots, n+2\}$ by

$f(x)=2 n-x$ one to one and onto function, see for example when $n=19$,

$$
\begin{gathered}
19+2=21 \leftrightarrow 19-2=17 \\
19+4=23 \leftrightarrow 19-4=15 \\
19+6=25 \leftrightarrow 19-6=13 \\
19+8=27 \leftrightarrow 19-8=11 \\
19+10=29 \leftrightarrow 19-10=9 \\
19+12=31 \leftrightarrow 19-12=7 \\
19+14=33 \leftrightarrow 19-14=5 \\
19+16=35 \leftrightarrow 19-16=3 \\
19+18=37 \leftrightarrow 19-18=1 \\
A=\{1,3,5, \ldots, 17\}, \quad B=\{37,35,33, \ldots, 21\}, \\
f: A \rightarrow B \text { defined by } f(x)=2 n-x .
\end{gathered}
$$

If $a=12$ then $19+12$ and $19-12$ are

simultaneously primes. Similarly when $n$ even number, we can define the one to one and onto function from the set

$C=\{1,3,5, \ldots, n-1\}$ to the set

$D=\{2 n-1,2 n-3,2 n-5, \ldots, n+1\}$ by

$f(x)=2 n-x$

\section{Proof of theorem (2.1)}

For every even integer $n \geq 4$, there exists a positive integer $a=n-p$ where $p$ is a prime number such that $3 \leq p \leq(n-1)$, makes $n-a$ is prime for all $p$, as well

$n+a=n+n-p=2 n-p$. We want to show that $2 n-p$ is prime for some $p$, such that $3 \leq p \leq$ $(n-1)$. Now let

$C=\{1,3,5, \ldots, n-1\}$, the set of all possible $n-a$, $D=\{2 n-1,2 n-3,2 n-5, \ldots, n+1\}$, the set of all possible $n+a$, and $f: C \rightarrow D$ defined by $f(x)=2 n-x$. In the both sets $C$ and $D$, there are primes numbers, we show by contradiction that $2 n-x$ can be prime number for some

prime no. $x$. Assume that $2 n-x$ is not prime number $\forall x=p$. That is $2 n-p \neq \bar{p}, \forall(\bar{p}$ prime number in the set $D)$.

Therefor $f^{-1}(2 n-p) \neq f^{-1}(\bar{p}), \forall \bar{p}$.

So that $\quad p \neq 2 n-\bar{p}, \forall \bar{p}$. Similarly for odd integer.

\section{Theorem (2.5)}

The necessary and sufficient condition of stronger Goldbach conjecture is theorem (2.1). 
Wildan .W

\section{Proof.}

Let $n \geq 2,2 n=p+q(p$ and $q$ are prime numbers).

We want to show that: $\exists a \in \mathbb{Z}^{+}$, s.t, $(n-a)$ and $(n+a)$ are simultaneously primes. We have $2 n-p=q$, there exists a positive integer $a=n-p$ such that $n-a=p$ and $n+a=q$.

Conversely, $2 n=n+n$, (if $n$ is prime number then $a=0)$. Then by theorem (2.1), $\exists a \in \mathbb{Z}^{+}$, s.t , $(n-a)$ and $(n+a)$ are simultaneously primes. Therefore

$$
2 n=n+n=(n-a)+(n+a) \text {. }
$$

\section{Reference}

[1] L.E.Dickson, "History of the theory of numbers",vol.1, New York, 1934.

[2] J.Richstein, "Verifying the Goldbach conjecture up to $4 \cdot 10^{14}$.", math.comp, 70:236,1745-1749, 2001

[3] H.A. Helfgott, "Major arcs for Goldbach's theorem". arXiv: 1305.2897, 2013.

[4] H.A. Helfgott, "Minor arcs for Goldbach's problem". $\underline{\text { arXiv: }}$ 1205.5252, 2012.

\section{اثبات لمخمنة غولاباخ الأقوى ( الثنائية )}

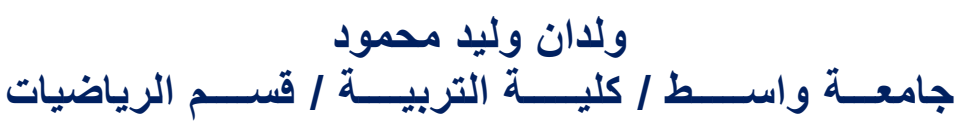

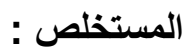

يعبر عن مخمنة غولدباخ الأقوى (الثنائية) بأنها " كل عدد صحيح زوجي أكبر من أو يساوي 4

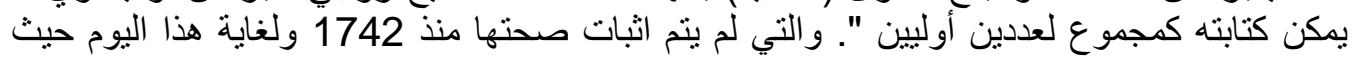

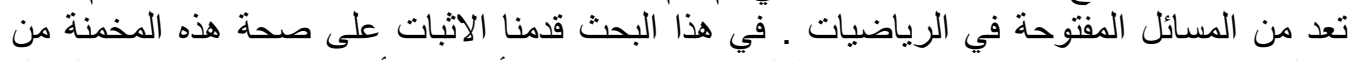

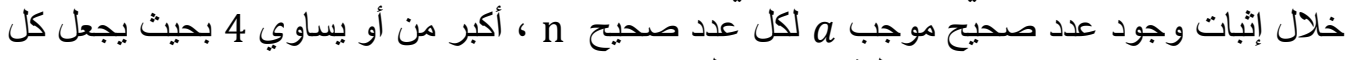

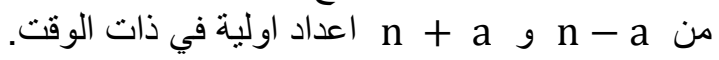

